\title{
Scanning Transmission Electron Microscopy Methods for the Analysis of Nanoparticles
}

\author{
Arturo Ponce, Sergio Mejía-Rosales, and Miguel José-Yacamán
}

\begin{abstract}
Here we review the scanning transmission electron microscopy (STEM) characterization technique and STEM imaging methods. We describe applications of STEM for studying inorganic nanoparticles, and other uses of STEM in biological and health sciences and discuss how to interpret STEM results. The STEM imaging mode has certain benefits compared with the broad-beam illumination mode; the main advantage is the collection of the information about the specimen using a high angular annular dark field (HAADF) detector, in which the images registered have different levels of contrast related to the chemical composition of the sample. Another advantage of its use in the analysis of biological samples is its contrast for thick stained sections, since HAADF images of samples with thickness of 100-120 nm have notoriously better contrast than those obtained by other techniques. Combining the HAADF-STEM imaging with the new aberration correction era, the STEM technique reaches a direct way to imaging the atomistic structure and composition of nanostructures at a sub-angstrom resolution. Thus, alloying in metallic nanoparticles is directly resolved at atomic scale by the HAADF-STEM imaging, and the comparison of the STEM images with results from simulations gives a very powerful way of analysis of structure and composition. The use of X-ray energy dispersive spectroscopy attached to the electron microscope for STEM mode is also described. In issues where characterization at the atomic scale of the interaction between metallic nanoparticles and biological systems is needed, all the associated techniques to STEM become powerful tools for the best understanding on how to use these particles in biomedical applications.
\end{abstract}

Key words: Scanning transmission electron microscopy, STEM, Inorganic nanoparticles, Biological systems, HAADF-STEM, Aberration-corrected microscopy

\section{Introduction}

Conventional transmission electron microscopy (TEM) is a beam characterization technique with almost 80 years since the development of the first microscope. In TEM, a large area of the specimen is illuminated, the magnification is performed by the lens system underneath the specimen, and subsequently the whole image is registered instantaneously. Conventional scanning electron 
microscopy (SEM) was designed after the TEM and it has been widely used for surface analyses in biological and materials sciences. The scanning-transmission electron microscopy (STEM) combines both operational modes. In STEM mode, a convergent electron beam is focused to a small area of the sample. To register an image, the electron probe is raster-scanned and subsequently propagated through the sample. Due to the electron-matter interaction, the trajectory of the electrons is scattered away and different kinds of signals are registered in sync with the electron probe scanning. The resolution of the STEM is mostly determined by the size of the probe. The newest electron microscopes can work in both modes, STEM or TEM, so there is a tendency to discard the use of dedicated instruments. One of the main advantages of the STEM over the TEM is that the signal generated by the electrons scattered out to high angles on a high-angle annular dark field detector (HAADF), is chemically sensitive, and a sample with a definite crystalline arrangement is not necessarily a requirement. The capacity of STEM of generating these different levels of contrast is commonly known as Z-contrast; the contrast dependance goes approximately as $\mathrm{Z}^{2}, \mathrm{Z}$ corresponding to the atomic weight of the element that caused the scattering of the electrons (1).

For nanostructures, TEM and STEM operational modes become necessary due to the fact that matter properties from bulk can result in different-sometimes even improved-properties at the nanoscale from those at bulk. This dependence in size is due to several reasons: quantum effects, surface effects, and modification of thermal behavior, among others $(2,3)$. In order to take advantage of the physical and chemical properties of the nanostructures by guiding the fine-tuning of these properties depending on the purposes for which the structures has been created, a deep understanding on the relation between shape, size, and function, is needed. The appropriate generation and interpretation of electron micrographs is crucial for this purpose. The interpretation of the electron micrographs is not always straightforward, since the intensity signal that correlates with the atomic positions depends not just in the length of the atomic columns parallel to the direction of the electron beam, but also on the chemical species, and on the microscope's parameters at which the micrograph was obtained (4). In this way, the problem of extracting a third dimension from the information contained in a strictly two-dimensional image requires the comparison of the observed images against simple models acquired by previous experience or inherited, and in many cases a theoretical model of the structure is necessary.

This chapter deals with the specific issues that need to be considered in order to obtain useful micrographs and to make an adequate interpretation of the micrograph of a nanostructure, with a particular emphasis in the imaging of nanoparticles used as good candidates to be incorporated in biomedical applications. We will 
concentrate in the use of STEM imaging. The chapter aims to be a guide for researchers interested in the characterization, at atomic scale, of metallic nanoparticles, and nanostructures. We will cover the working principles of STEM, the role of aberration correctors, and how the theory supporting the imaging of micrographs can be used to simulate the imaging process, to concentrate later on the practical issues concerning the comparison and interpretation of real and simulated electron micrographs of nanostructures.

\section{Stem Imaging}

In TEM, the information transfer in phase contrast imaging is determined by the objective lens and the size of it aperture, which are directly related to the temporal and spatial coherence of the electrons. In Fig. 1, a conventional high resolution transmission electron microscopy (HRTEM) micrograph of a bimetallic nanoparticle of $2 \mathrm{~nm}$ of diameter shows the interatomic distances; however, the contrast is not directly related with the different atomic positions in the bimetallic nanoparticle (5) proposed by the theoretical model shown in Fig. 1b. The optical arrangement of a conventional TEM compared with the STEM configuration is illustrated in the schematic diagrams shown in Fig. 2. The scattered electrons in STEM mode can be registered by three different detectors. The bright field $(\mathrm{BF})$ detector collects the electrons transmitted in the path of the beam close to the optical axis, the BF electrons containing the total beam current. The annular dark field (ADF) and the HAADF detectors are used to record the electrons scattered out of the path of the beam. From the point of view of
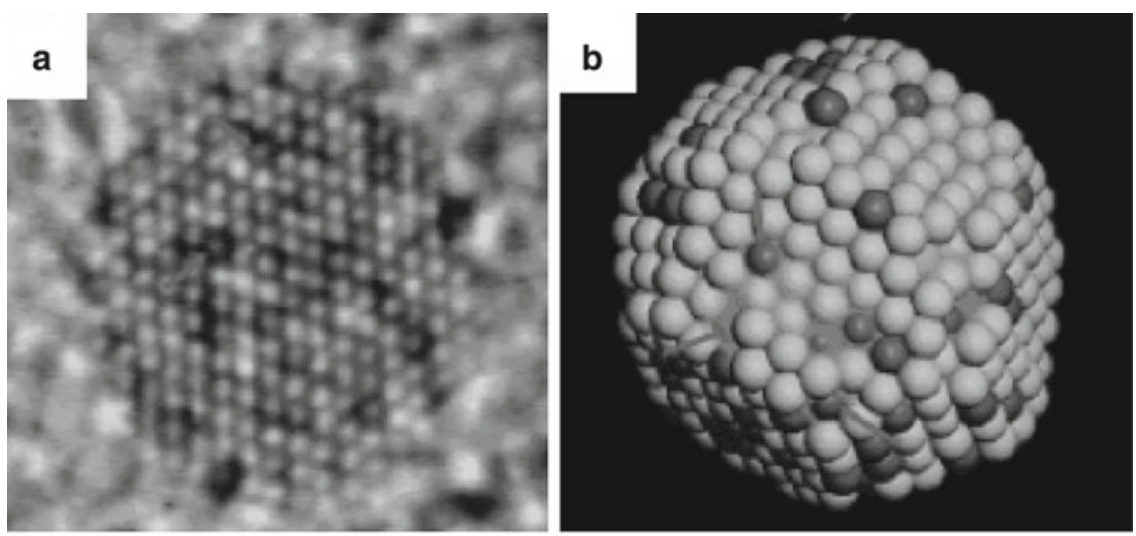

$5 \mathrm{~nm}$

Fig. 1. Conventional high resolution transmission electron microscopy (HRTEM). (a) HRTEM image of a bimetallic goldpalladium nanoparticle. (b) Theoretical model of the bimetallic nanoparticle. 

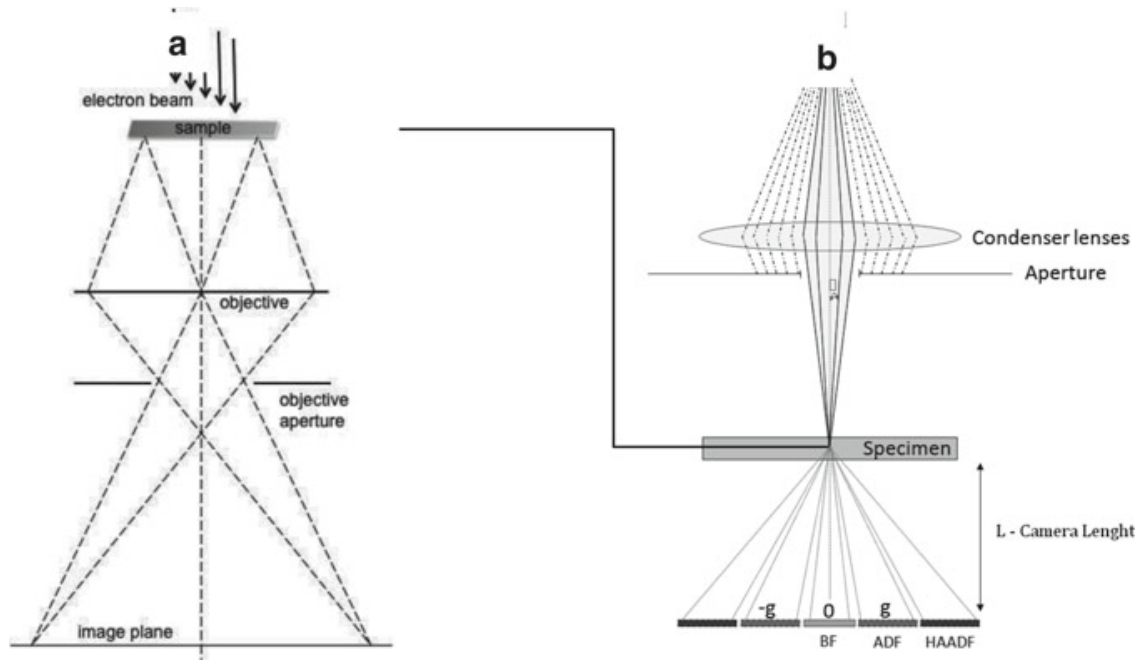

Fig. 2. Optical arrangement of a conventional transmission electron microscopy (TEM) and scanning transmission electron microscopy (STEM) configurations. (a) TEM and (b) STEM operational modes.

the reciprocity theorem, the STEM is optically equivalent to an inverted TEM, in the sense that if the source and the detector exchange positions, the electron ray paths remain the same (6). In the STEM, the objective lens-and all the relevant optics-are positioned before the specimen. The scattered angle of the ADF detector is set around $\sim 40 \mathrm{mrad}$, and the outer angle is set around 60-200 mrad (see Fig. 2). STEM differs from conventional TEM in that the electron beam interacts only with a small section of the sample; the scanning process is the one in charge of generating the image as a whole, while in TEM, a broad beam is interacting instantaneously with the sample. X-ray energy dispersive spectroscopy (EDS) attached to the STEM mode provides with an elemental analysis directly from the point or line raster-scanned in the sample. Therefore, the imaging using the BF or HAADF detectors in a complementary way can be matched to the EDS information, and the elemental mapping at atomic resolution is obtained. Figure 3 shows the setup of the STEM mode including the detectors a set of the detectors typically used in STEM mode. Both the HAADF and BF detectors are shown in the picture. Examples of HAADF and BF images are included in Fig. 3 (on the right); both can be acquired simultaneously. $L$ corresponds to the camera length, which is the effective distance or magnification between the specimen and the detector plane position. The X-ray detector is also shown in Fig. 3; the spectra can be collected at the same time together with STEM images. An example of the use of EDS and HAADF/BF-STEM is illustrated in Fig. 4, where a set of 


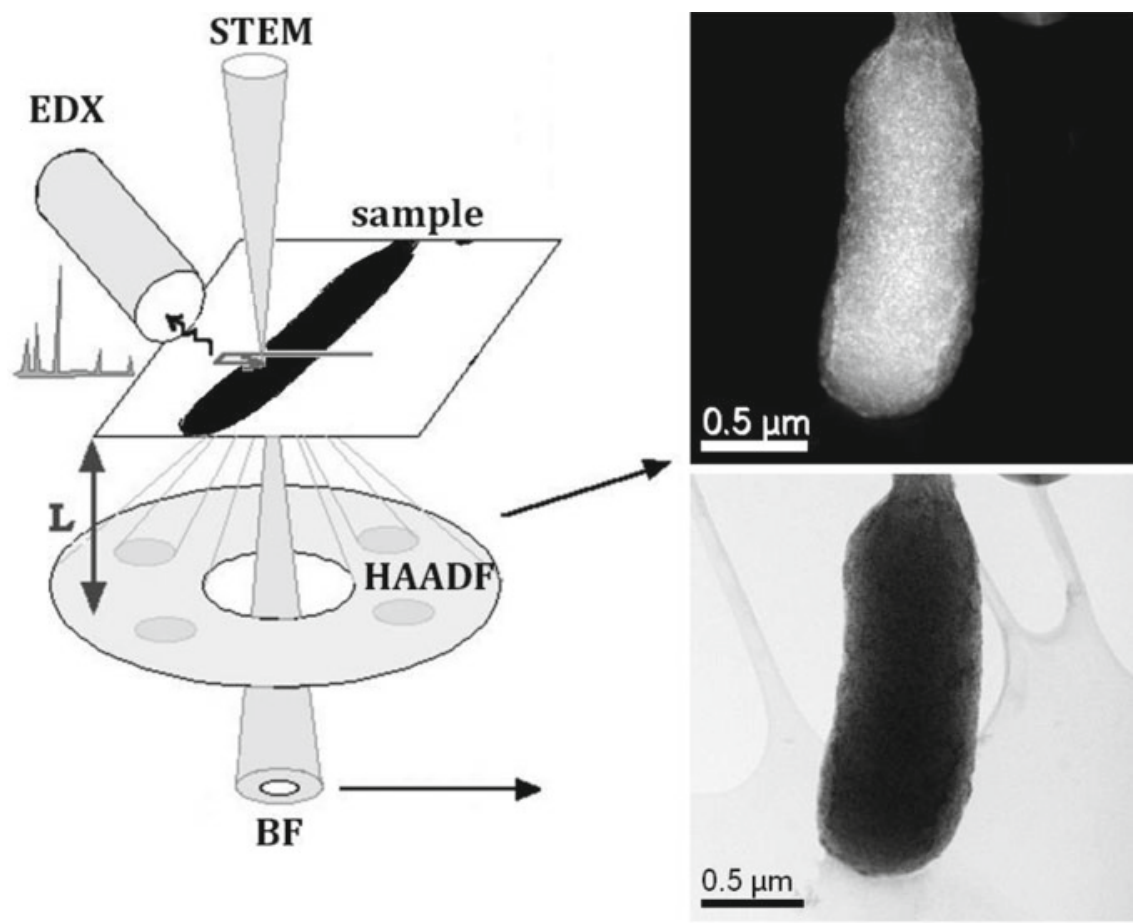

Fig. 3. STEM setup including the energy dispersive spectroscopy (EDS) spectrum detector.
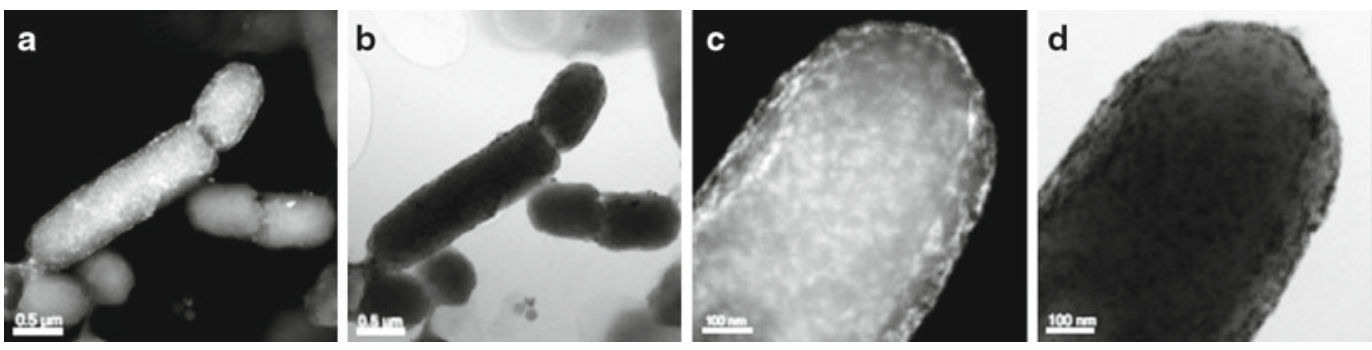

Fig. 4. STEM imaging of Escherichia coli bacteria. (a, c) high angular annular dark field (HAADF)-STEM, (b, d) BF-STEM.

STEM images from a bacterium show high contrast due to the presence of terbium inside and in the periphery of cells, which confirms the incorporation of $\mathrm{Tb}$ and indicates that TATA-binding protein (TBP) was mainly located in the periplasmic region. For HAADF images, the high contrast corresponds to the $\mathrm{Tb}$ incorporation in the bacteria. The EDS spectrum obtained in a transversal region of bacteria is shown in Fig. 5 . 

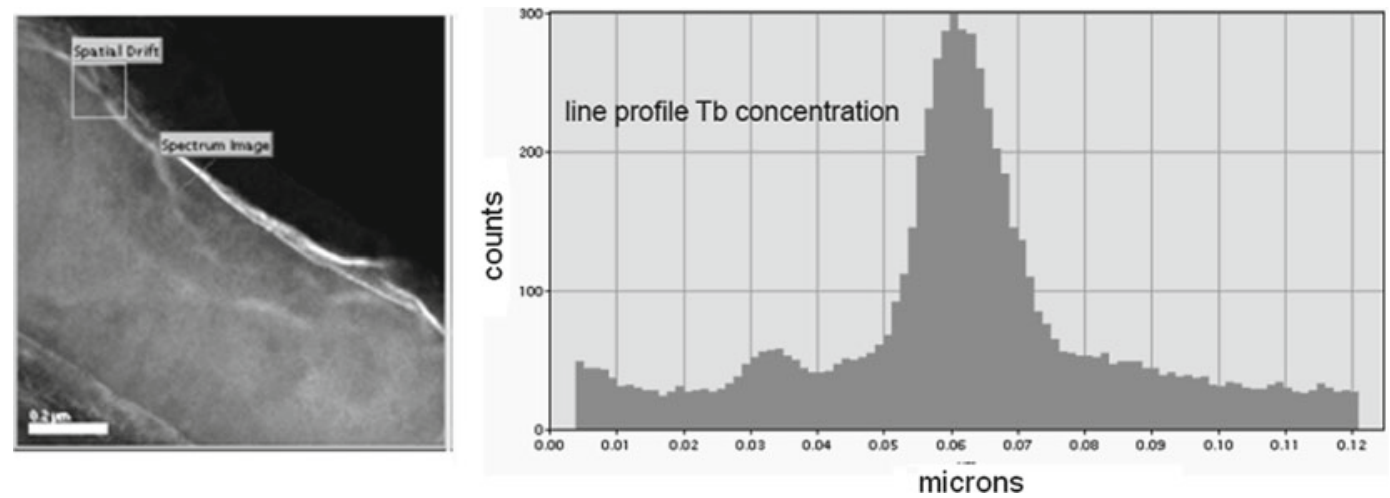

Fig. 5. Line profile EDS spectrum. STEM image showing transversal region analyzed (left). Tb line profile of transversal region analyzed (right).

\section{Practical Considerations for Stem Imaging}

The basic parameters described in this section are used to register high resolution STEM images. The process must be initialized in TEM mode for searching field of view and adjust the crystal orientation if necessary. The eucentric focus must be adjusted with the z-control. By following the TEM initial setup, the system can be switched to STEM. The illumination focused on the sample must be adjusted by the size of the condensed aperture, spot size, and camera length $(L)$. The camera length is the effective distance or magnification between the specimen and the detector plane position (labeled in Fig. 2). The collection angle depends on the microscope camera length. Typical values for ADF and HAADF detectors vary from $\sim 40$ and $200 \mathrm{mrad}$, respectively.

The resolution in STEM depends upon the spot size of the electron beam. Both spot size and the aperture of the condenser lens have influence in the beam current density in the sample and as consequence, in the resolution of the instrument. The beam current density in the sample can be increased by a large aperture of the condenser lens or by using a lower spot size. Figure 6 shows two different settings for STEM. Increasing the spot size (spot number) leads to increased demagnification of the source and a decreased current in the beam. Increasing the spot number leads to a reduction of the beam current. Changing the size of the condenser lenses CLl and CL2, produces that the beam current is also changed. In Fig. 7, the sketches for the rays using two different sizes of the condenser apertures are illustrated. The selected aperture must be centered in the Ronchigram (described in next section) as is shown in Fig. 7. Spot size and aperture of the 


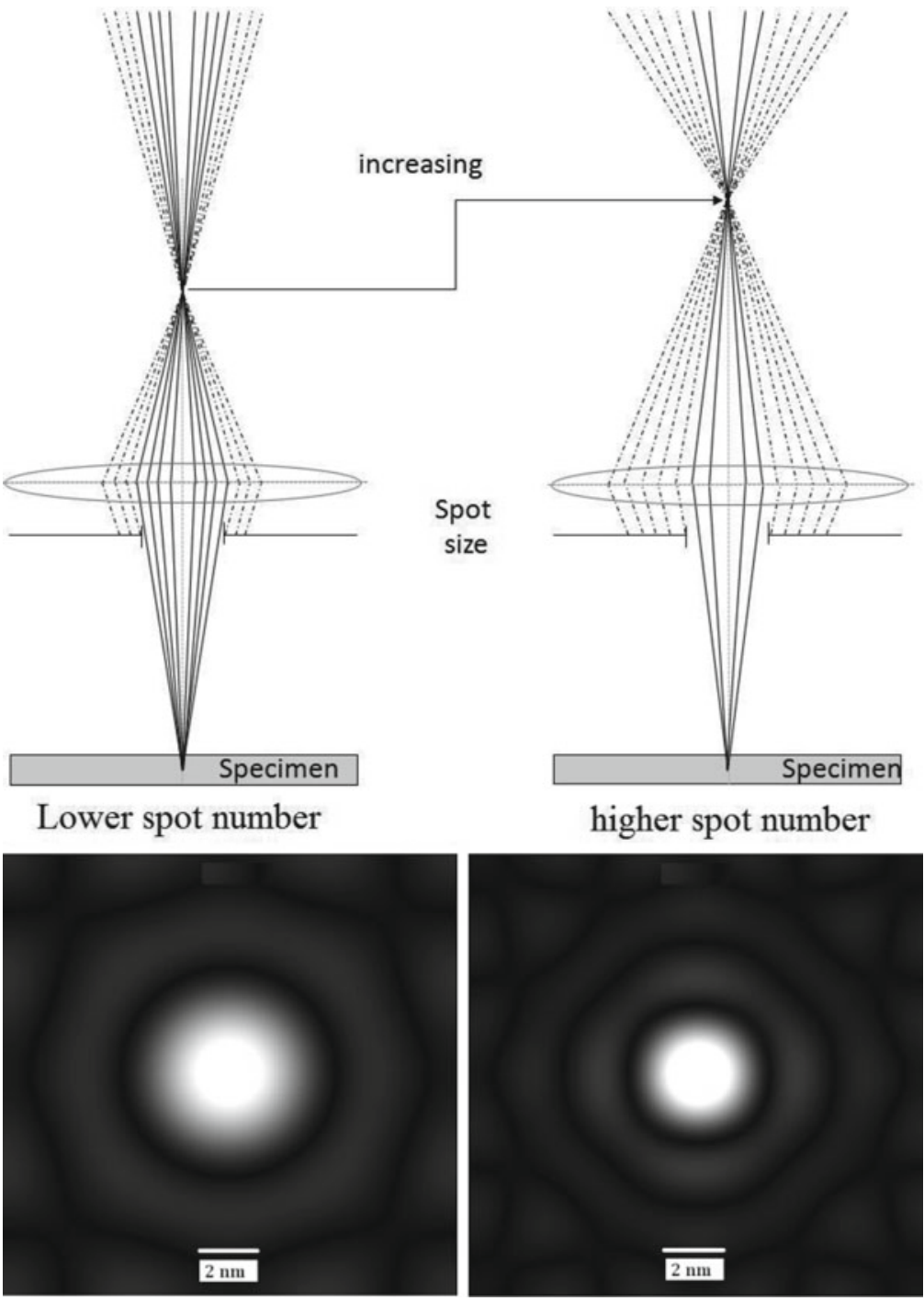

Fig. 6. Electron beam sketches varying the spot size and the potential projected simulated depending on the spot size. Smaller spot size (left), larger spot size (right).

condenser lens determine the resolution of the microscope in STEM mode. The spot size, measured in nanometers, can be simulated as a function of the parameters of the microscope (7).

\section{The Ronchigram}

A Ronchigram is actually a shadow image, referring to the features at the center of the convergent beam electron diffraction (CBED) pattern, and formed by a focused and stationary electron probe on 

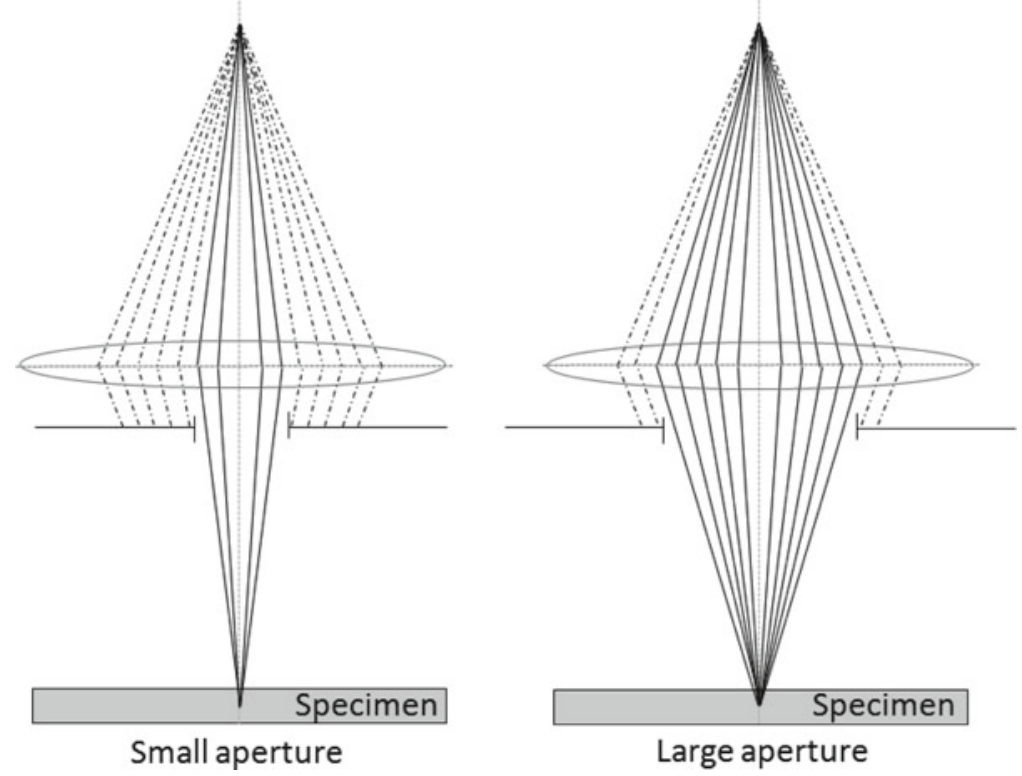

Fig. 7. Electron beam trajectories by varying the condenser lens apertures. Smaller aperture (left), larger aperture (right).

amorphous material. The quality and resolution of the STEM images depend directly on the proper alignment of the Ronchigram. It is easily observable in field emission gun (FEG) microscopes, but it might be difficult to obtain in the Lanthanum Hexaboride $\left(\mathrm{LaB}_{6}\right)$ filaments because the effective probe size is too large, and therefore both the spatial resolution and the brightness decrease dramatically, even using microscopes with voltages higher than $200 \mathrm{kV}$. All the parameters used to adjust different sets of contrast, spatial resolution, etc. are directly related with the Ronchigram. The Ronchigram can be registered in a CCD camera. The electrons coming out of the sample can be observed directly in a circle called the Gabor hologram, the Ronchigram or the central zero-order disk of the CBED pattern. The Ronchigram from an amorphous region should look as in the Fig. 8. The Ronchigram is actually a shadow image, referring to the feature within the central disk of a CBED pattern and formed by a small, focused, and stationary probe on thin amorphous region. The optimal defocus is useful to optimize the spatial resolution and it can be corrected from the Ronchigram. When a near-focused beam illuminated on the specimen, the Ronchigram changes with the illumination conditions. In under and overfocus, an image of the sample is observed. Overfocus, infocus, and underfocus Ronchigrams and the beam incident on the sample are illustrated in Fig. 9. The shape of the Ronchigram can be simulated using the parameters of the microscope (7). 

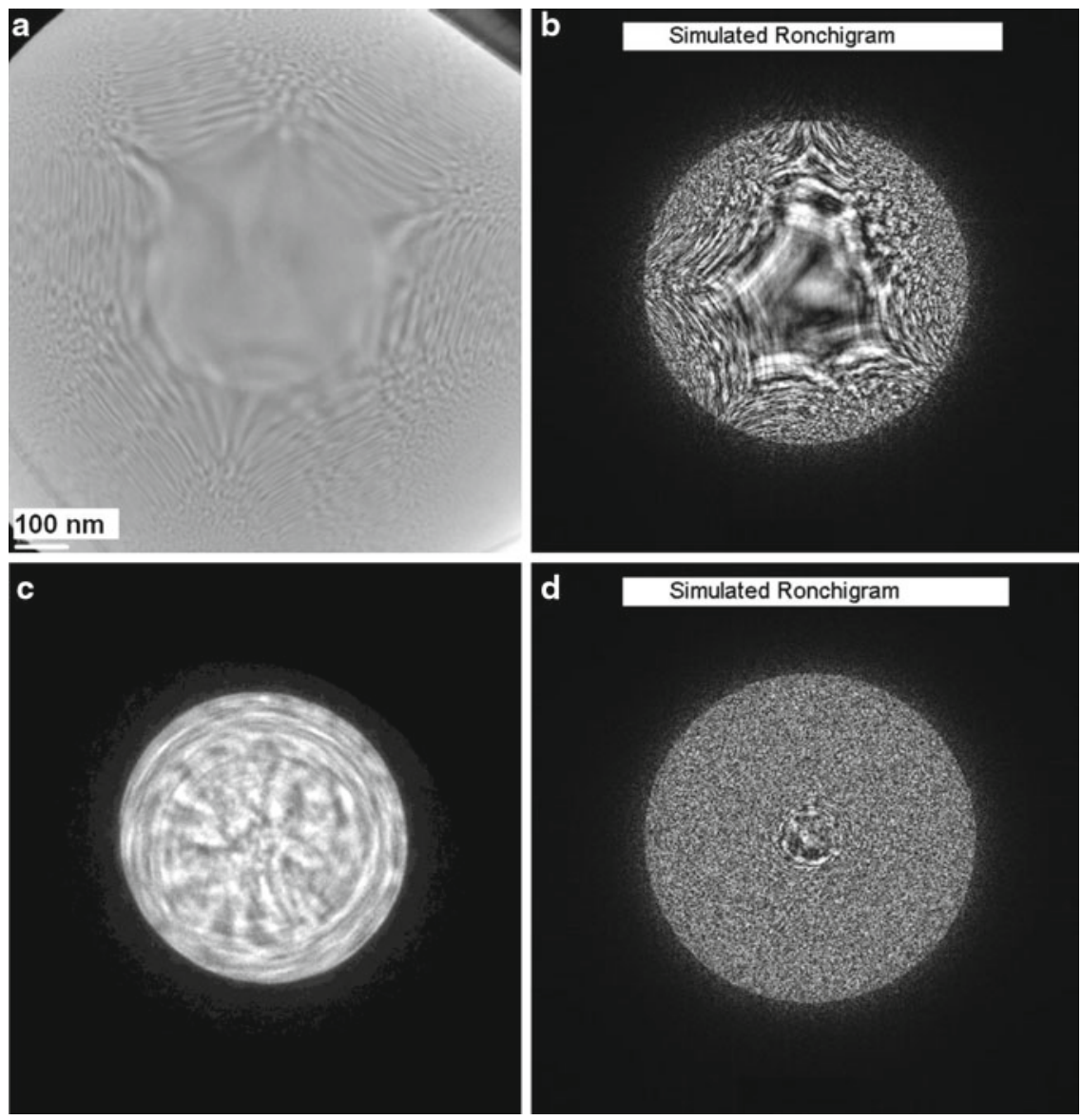

Fig. 8. Ronchigram images. Aberration-corrected experimental Ronchigram from an amorphous material (a); simulated Ronchigram (b) (7). Not corrected experimental Ronchigram from an amorphous material (c) and simulated Ronchigram (d) (7). The Ronchigram shown in (a) was obtained in an aberration-corrected microscope Jeol ARM200F, at the Kleberg Advance Microscopy Center at UTSA.

Electron microscopy has been improved greatly since 1990s with the correction of the spherical aberration and the chromatic aberration and is now capable of sub-angstrom resolution (1). Another important aberration to be corrected is the axial coma. The coma astigmatism occurs when the direction of illumination does not coincide with the direction of the true optical axis of the electron microscope. To reduce the effect of comma aberration in STEM, the beam must to be centered in the center in underfocus and overfocus conditions. The center of the Ronchigram must be positioned at the same point. Misalignment in $X$ and $\Upsilon$ direction can be observed in the Fig. 10 . The corrected $X$ and $\Upsilon$ directions of the comma astigmatism are observed in the center of the Ronchigram illustrated in the Fig. 10. Astigmatism of the condenser lens must be aligned in $X$ and $\Upsilon$ directions. Non astigmatic Ronchigram and induced $X$ and $\Upsilon$ astigmatism are shown in Fig. 11 . 


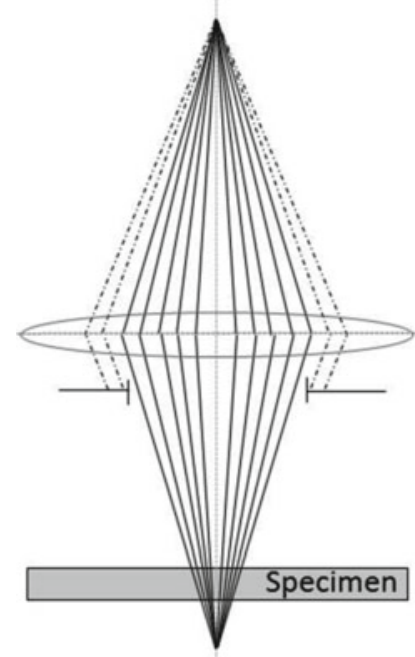

Underfocus

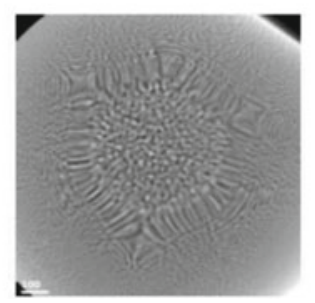

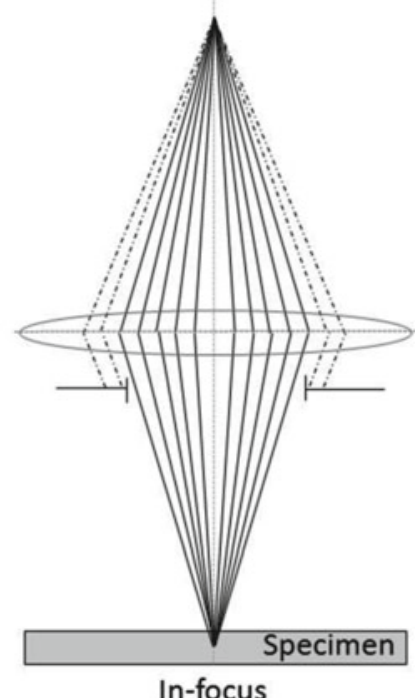
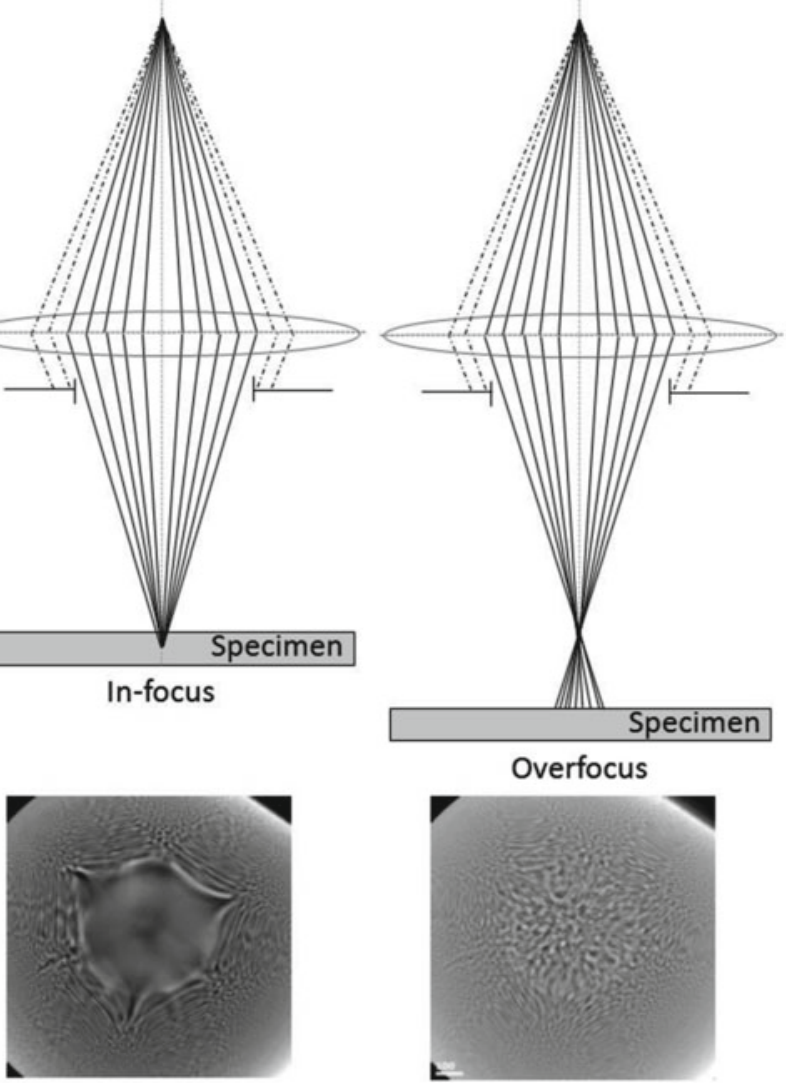

Fig. 9. Ronchigrams recorded with an underfocus, optimal defocus and overfocus electron probe on an amorphous specimen.

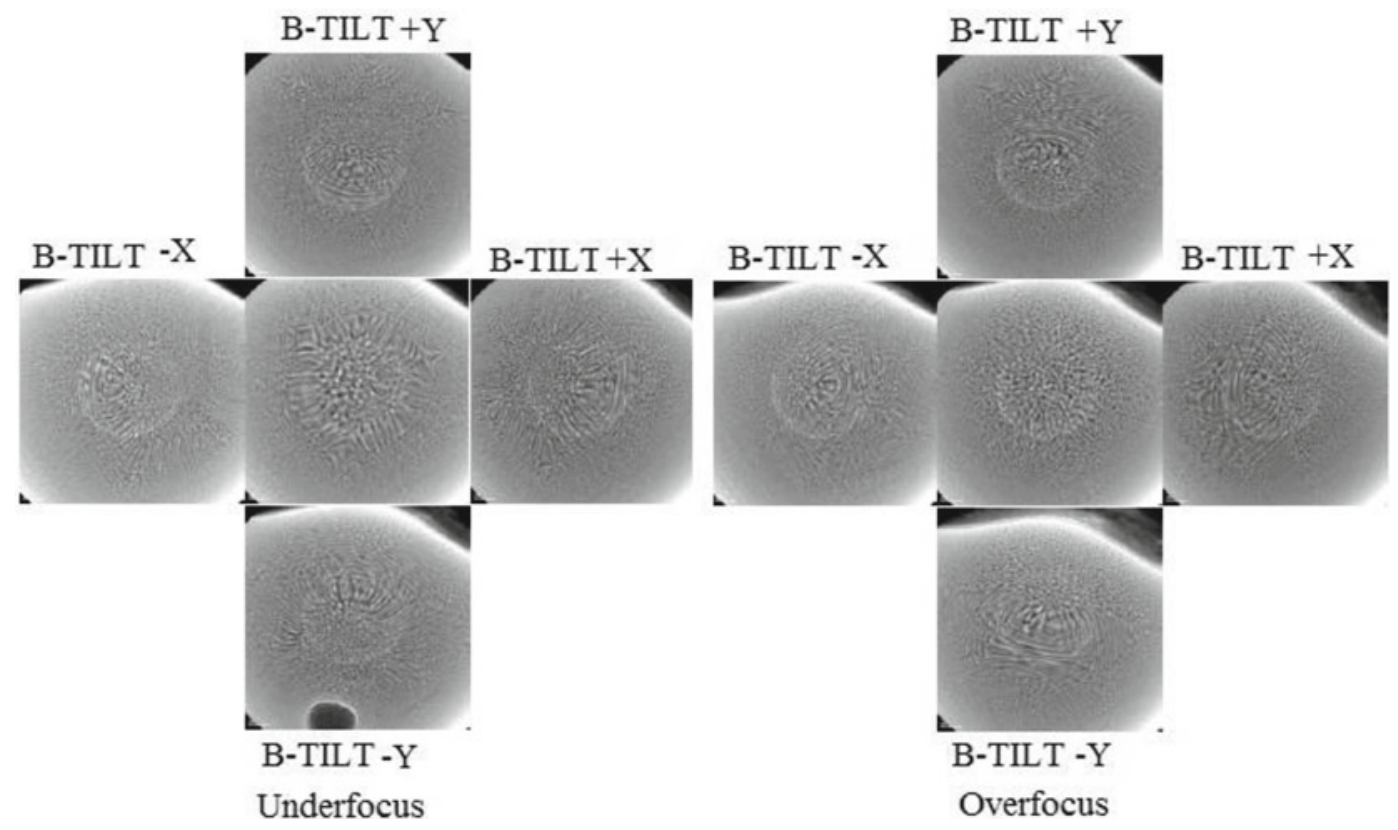

Fig. 10. Correction of the axial coma (beam tilt). 
a

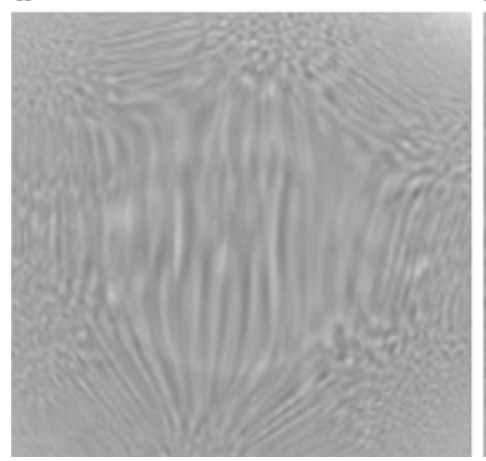

b

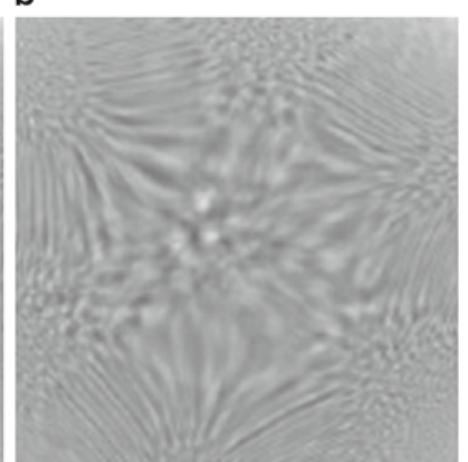

C

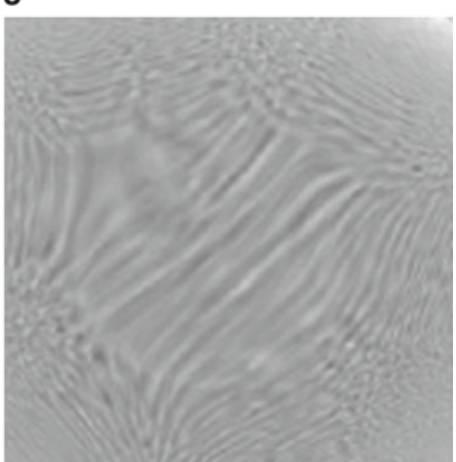

Fig. 11. Correction of the astigmatism in STEM. Induced astigmatism by $x(\mathbf{a})$; no astigmatism (b); induced astigmatism by $y(\mathbf{c})$.

\section{Atomic Number (Z) Dependence in HAADF-STEM Imaging}

HAADF image resolution is primarily determined by the size of the illumination probe, which is formed by the condenser system of the microscope. The best probe is formed at a balance of resolution (narrow illumination peak) and contrast (minimal tails), which means aperture semi-angle of $(4 \lambda / \mathrm{Cs})^{1 / 4}$ and defocus of $-(\lambda /$ Cs $)^{1 / 2}$. The resolution of incoherent STEM and TEM image will then be:

$$
\begin{aligned}
& \text { STEM }: \rho=0.43 \mathrm{Cs}^{1 / 4} \lambda^{3 / 4} \\
& \operatorname{TEM}: \rho=0.65 \mathrm{Cs}^{1 / 4} \lambda^{3 / 4}
\end{aligned}
$$

In this way, using the same energy in the microscope (same wavelength, $\lambda$ ) and the same spherical aberration (Cs), the STEM images are considerably better than TEM images, the spatial resolution is improved. It has been known for long time that the annular detector in a HAADF-STEM microscope will collect a large amount of the elastic scattered electrons, in such a way that the intensity of the signal collected by the detector will have a dependence on the scattering cross-section, and thus on the atomic number of the atoms in the sample (8). Pennycook mentions a dependence close to $Z^{3 / 2}(1)$. For a single atom, the distribution of electrons being scattered is more or less like a Gaussian function (Rutherford scattering). In order to confirm how the intensity signal generated in the HAADF detector depends on the atomic number of a column formed by just one atom of a specific element, we performed a simulation of HAADF intensities in 16 different atoms, which have been projected in a 2D plane (Fig. 12). 


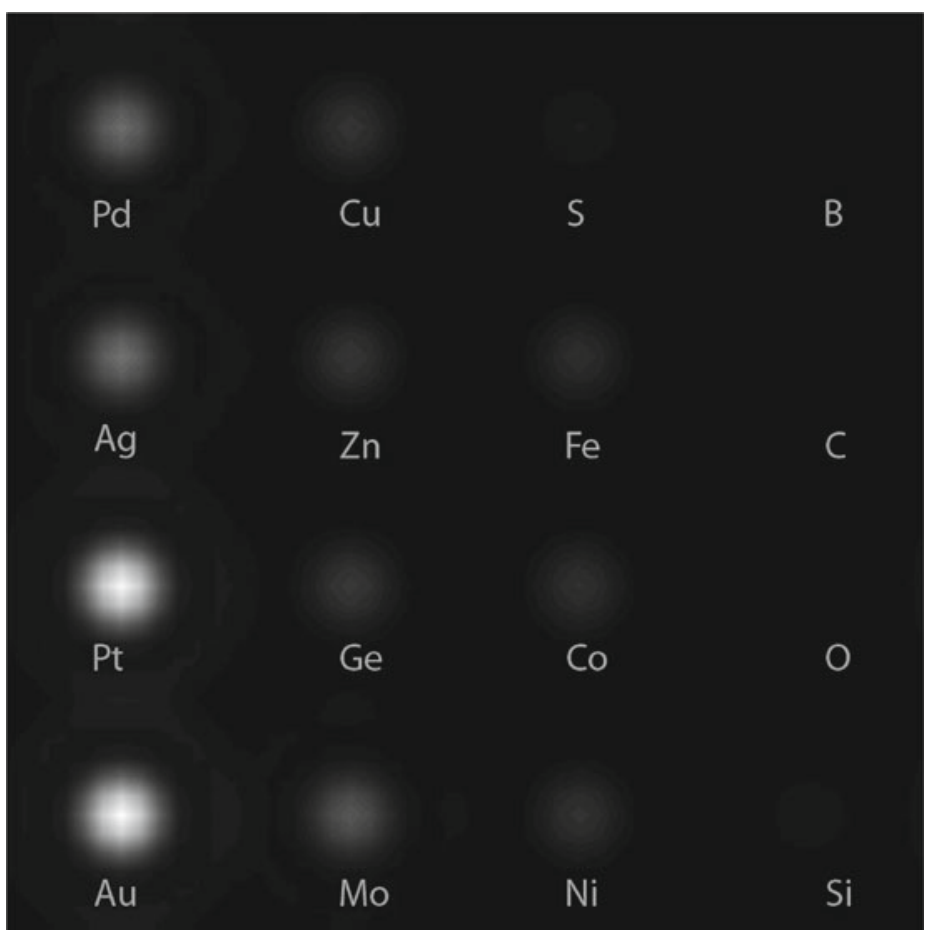

Fig. 12. Calculation of the relative HAADF-STEM contrast of different elements. Positions of the atoms are labeled by the name of their respective chemical element.

The line scan of the HAADF image intensity through the center of the atoms is shown in Fig. 13, where the high contrast between heavy and light atoms can easily be noted. The heights of the maxima from Fig. 13 are plotted against the relevant atomic numbers in Fig. 14. The extrapolation adjustment shows that the HAADF signal varies as approximately $Z^{1.46}$ in a good agreement with the previously reported $Z^{3 / 2}$ (1). STEM imaging simulation can be obtained by using the commercial version of the xHREM package, designed and implemented by Ishizuka (9). The simulation process can be carried out using the Multislice method. The basic idea underlaying the multislice method is that the potential of the sample can be approximated by defining a number of slices of thickness $d z$, and projecting the potential due to the atoms of a particular slice to the central plane of this slice. Thus, a solution to the electron wavefunction is obtained for one slice, and used as input for the calculation of the next slice. This means that the choice of very thick slices will speed up the calculations, but the potential function will be poorly approximated. On the other hand, the use of very thin slices will improve the calculation of the projected potential, but the errors due to approximations will accumulate and undermine the final result. The appropriate choice of the number of slices is an issue that requires a generous amount of 


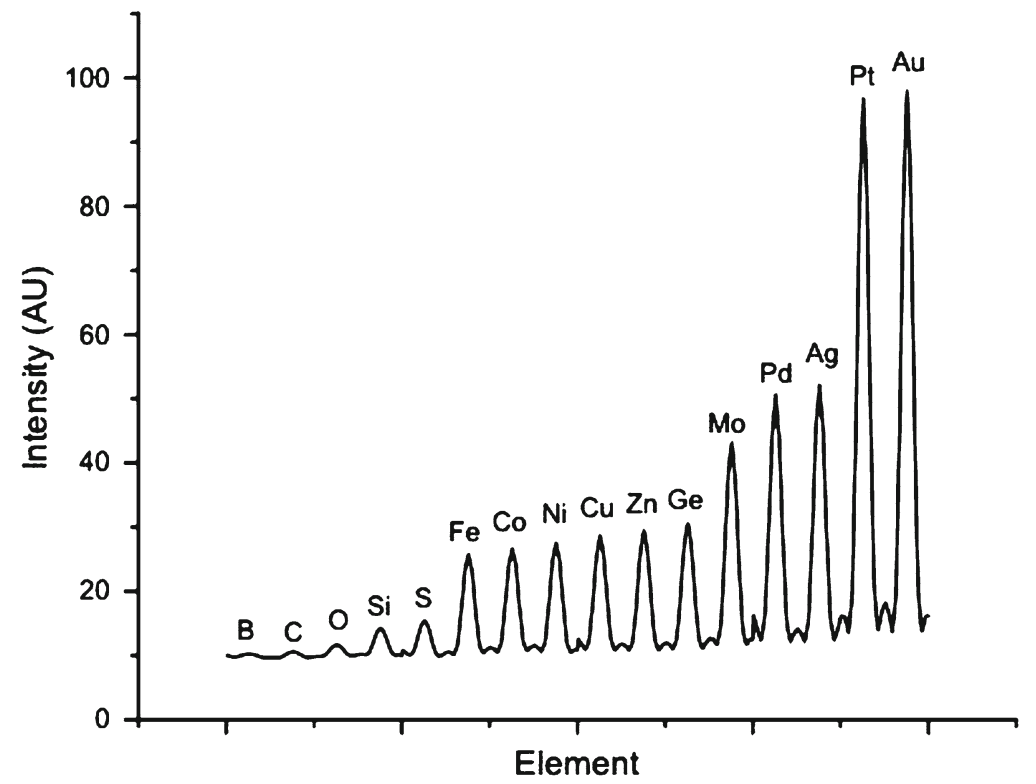

Fig. 13. HAADF-STEM Intensity signal profile relative to the chemical species, for the simulated STEM image shown in Fig. 12.

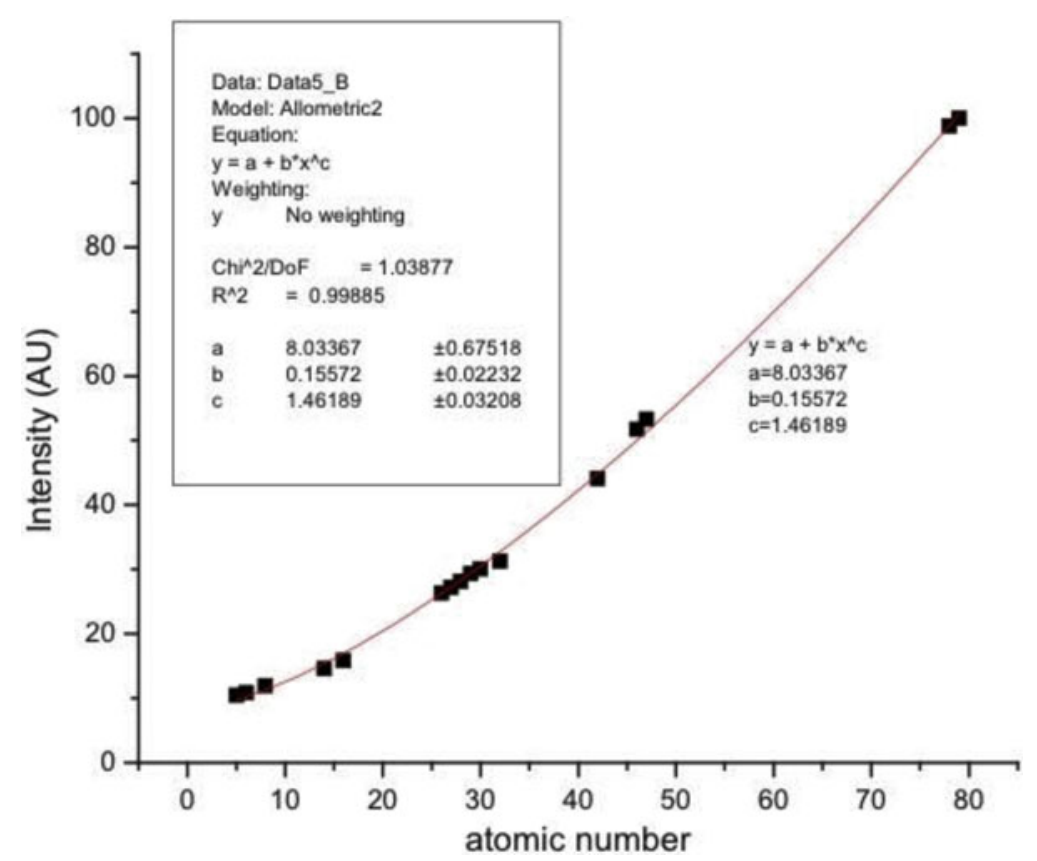

Fig. 14. Variation of the intensity of the contrast as a function of the atomic number.

effort and computing time, and the final decision must consider the benefits of a fast calculation (STEM simulations by the very nature of the technique are computationally expensive and the computing time depend both on the number of slices and the scan 
resolution) against the benefits of a very precise calculation (that may be critical in quantitative STEM). As pointed out by Ishizuka (9), the multislice method requires several approximations that could be critical in the interpretation of results for inclined illumination, so the orientation of the sample may also affect the final result. XHREM uses an algorithm based on Fast Fourier Transforms, and it allows the use of a large number of slices.

\section{Interpretation of Stem Images}

How can we infer the shapes, sizes, and composition of the objects studied under the electron microscope? The problem of interpretation of electron micrographs is similar to the problem that the human mind has to solve to make a correct interpretation of the two-dimensional images imprinted on the retina. As the linguist Steven Pinker puts it: "Optics is easy but inverse optics impossible" (10). The solution to this factual impossibility lays on the capacity of the human mind to make educated assumptions about the observed objects and their surroundings. The interpretation of the electron microscope images must be made under the same kind of assumptions: As a first approximation, one considers that the nanostructures are laying on an even surface (usually carbon), and that in inner regions of the nanostructure and far from twin boundaries, point-defects and dislocations, the atomic arrangement is close to that of the bulk.

As it was mentioned before, the approximations used in the different simulation techniques are strictly true only when the sample is thin enough for the linear assumption to be correct. In the multislice method the sample is dissected into several slices, each treated independently through an averaged projected potential. The selection of the number of slices is a tricky job, and it will depend on the thickness of the sample (the size of the nanostructure in the case of metal nanoparticles), the orientation, and the computational capabilities. The projected potential is calculated for each slice, and the corresponding output wavefunction is obtained. If the number of slices is increased, the number of projected potentials will increase as well. This will reflect on the final result of the image, as can be seen in Fig. 15, where a model of an icosahedral gold particle was used to generate two simulated STEM micrographs, being the only difference between the two number of slices, 10 slices (Fig. 15b), 20 slices (Fig. 15c). It is not a simple task to find out which of the images follows the experimental one in a better way, since the orientation and lattice parameters must be considered.

In the remaining of this chapter, for purposes of comparison we will restrict ourselves to use ten slices, irrespective of the model being simulated. We invite the reader to dedicate a generous amount of time to try out different possibilities. 

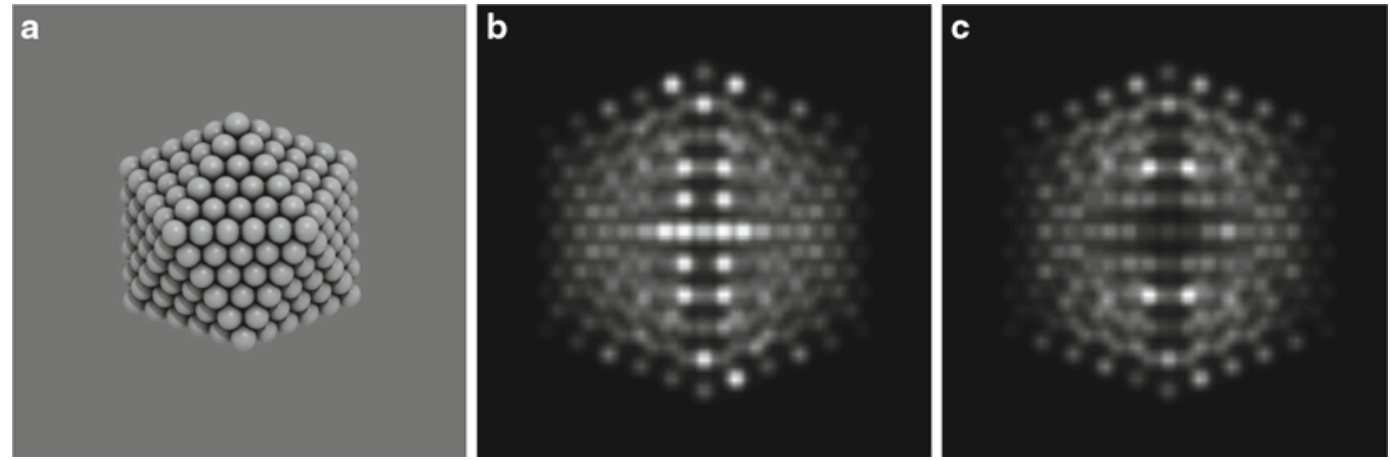

Fig. 15. Simulated STEM for icosahedral gold particle. Icosahedral gold particle model (a) and HAADF-STEM simulated images using 10 slices (b) or 20 slices (c) in the simulation.
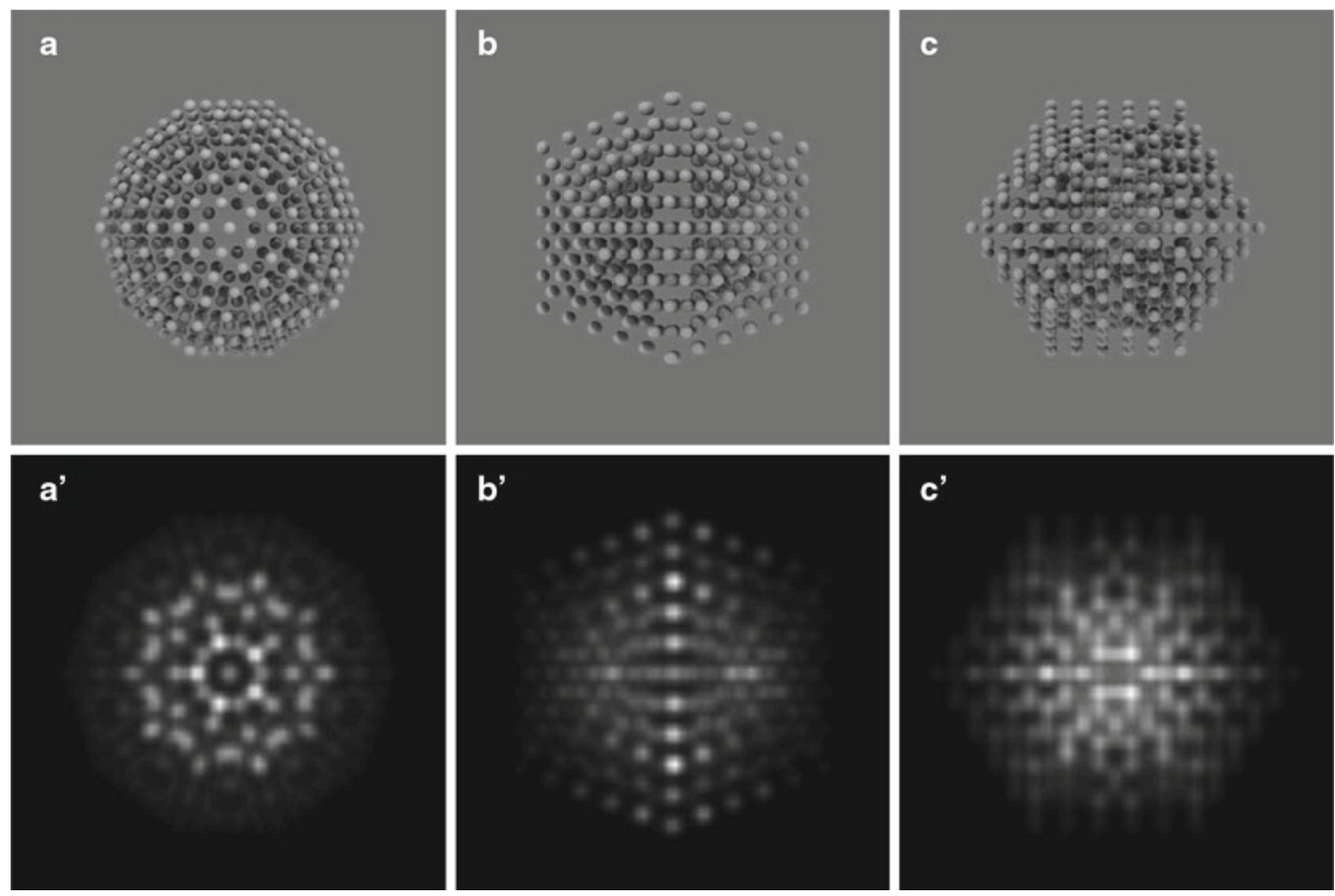

Fig. 16. Simulated STEM images of an icosahedral $A u_{\text {core }}-P d_{\text {shell }}$ particle. Core-shell structure model $(\mathbf{a}-\mathbf{c})$ and simulated STEM images $\left(\mathbf{a}^{\prime}-\mathbf{c}^{\prime}\right)$, at three different orientations.

Returning back to the issue of the Z-contrast dependency on STEM, we take advantage of the previous figure to compare it against the simulated STEM micrographs of particles of the same size and shape, but with different composition. Figure 16 shows the STEM simulated images of an icosahedral $\mathrm{Au}_{\text {core }}-\mathrm{Pd}_{\text {shell }}$ particle. The orientation shown in Fig. $16 \mathrm{~b}$ is rotated $30^{\circ}$ with respect to the orientation shown in Fig. 16a; the orientations shown in 

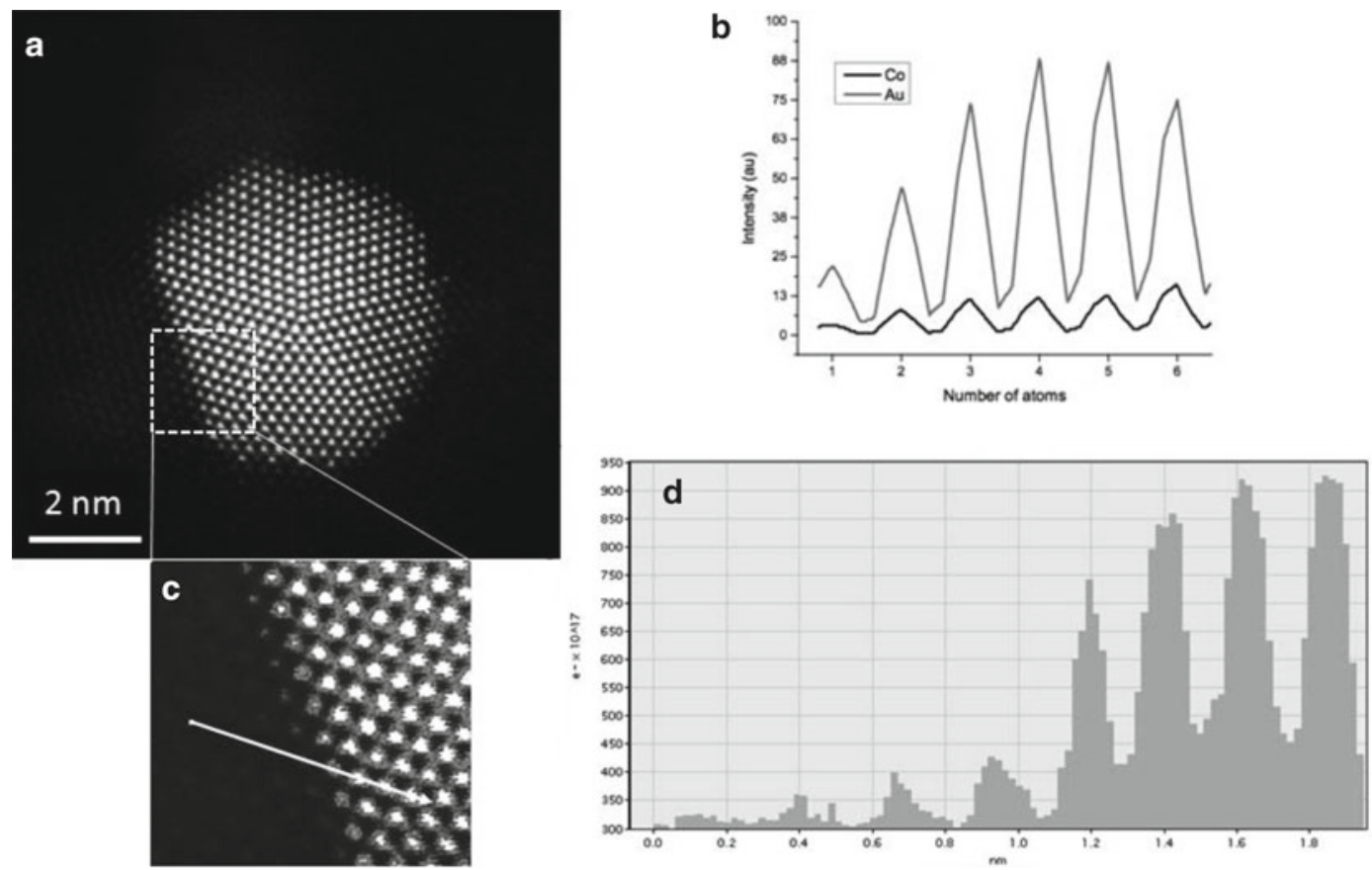

Fig. 17. Z-contrast capabilities of HAADF-STEM. Cs-corrected aberration HAADF-STEM image from Au/Co bimetallic nanoparticle (a). Calculated intensities profiles from Co and Au atoms (b). Enlarge Cs HAADF-STEM image (c) and experimental intensity profile including the $\mathrm{Co}$ and Au atomic spacing (d).

Fig. $16 \mathrm{c}$ is rotated $90^{\circ}$ with respect to Fig. 16a. Figure $16 a^{\prime}-c^{\prime}$ show respective simulated images. The difference in intensity between core and shell are evident. How can the Z-contrast capabilities of HAADF-STEM be used in the investigation of real metal nanoparticles? As an example, consider the Au-Co particle presented in Fig. 17a. This particle was produced by the coalescence of a gold particle with a cobalt particle, both originally synthesized by inert gas condensation and deposited on a carbon substrate. After deposition, the system was subjected to a heating process. The particle in Fig. 17c shows an enlargement to remark the differences in intensity, as well as the intensity profile in Fig. 17d. The two intensity profiles plotted in Fig. 17b show the simulated intensity profiles, described in Fig. 14. This result exemplifies the use of STEM simulations for an approximate description of composition and shape of metal nanoparticles.

\section{Examples of Stem-Imaging of Metallic Nanoparticles}

Using lower aperture of the condenser lens, spot number higher than 6 , the beam current density incident in the sample would be less than $30 \mathrm{pA}$. Under these conditions and the alignment 

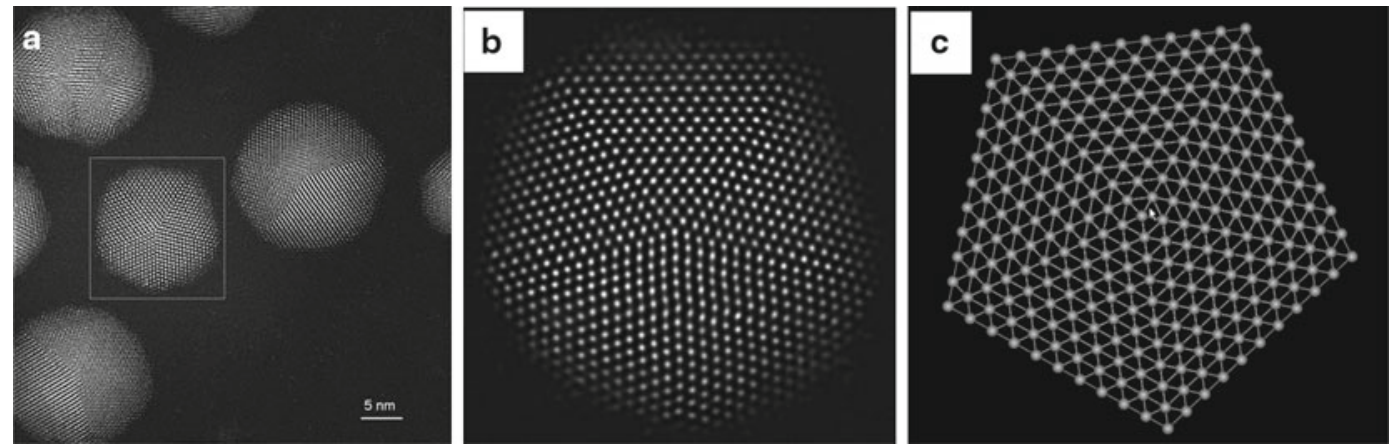

Fig. 18. Gold nanoparticles at atomic scale resolution. STEM image showing a few different gold nanoparticles (a); high magnification of a decahedral nanoparticle (b) and theoretical model of this decahedral nanoparticle (c).

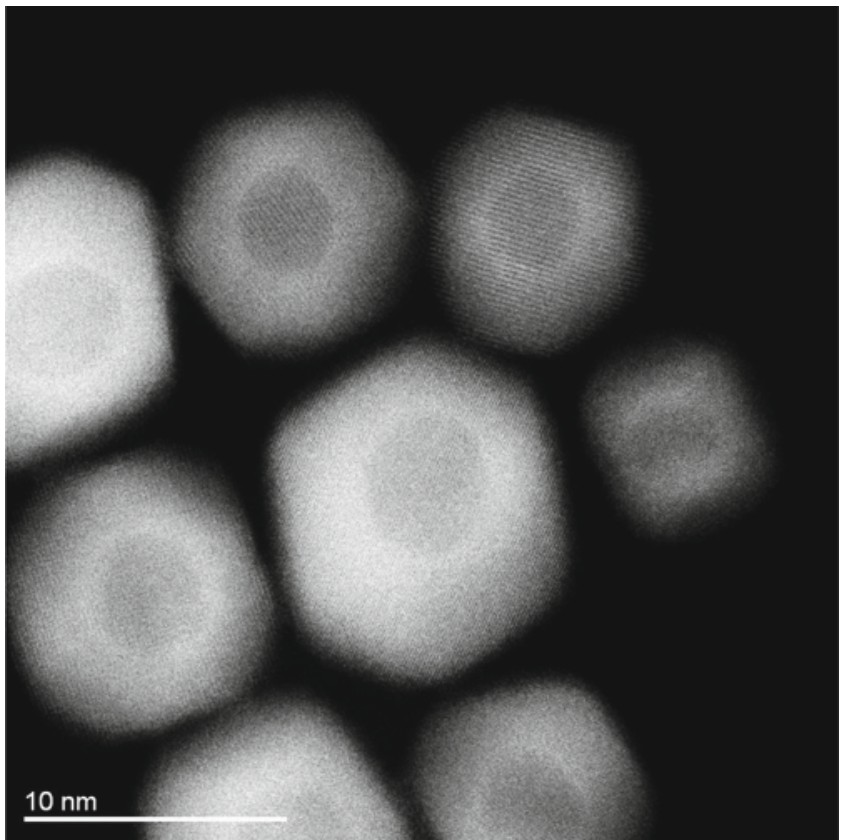

Fig. 19. Au/Pd core-shell structures obtained by HAADF-STEM.

mentioned in the previous sections, the high resolution STEM is guaranteed. Decahedra gold nanoparticles can be identified at atomic scale in the Fig. 18. Gold-palladium ( $\mathrm{Au} / \mathrm{Pt})$ bimetallic Core-shell structures can be identified by differences in the contrast in the HAADF-STEM imaging in Fig. 19. The high contrast corresponds to the gold shell (Au with $\mathrm{Z}$ atomic weight 79) whereas the inner core showed lower contrast corresponding to a core of palladium ( $\mathrm{Pt}$ with $\mathrm{Z}$ atomic weight 46 ).

The controlled incorporation of the metallic nanoparticles into biological systems is a challenge for the experimental scientist. The STEM imaging becomes as a critical tool to identify the incorporation and the influence of the multiple shapes into these biological 


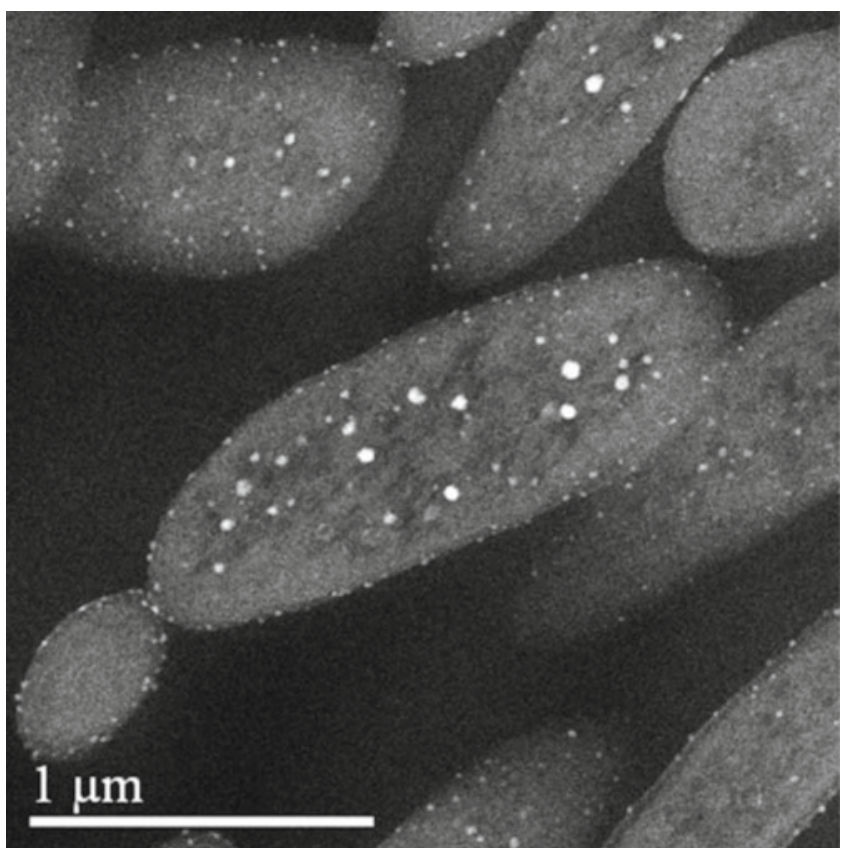

Fig. 20. HAADF-STEM image of silver nanoparticles incorporated into bacteria E. coli.

systems. Biologic systems contain mainly carbon, nitrogen, hydrogen as the matrix. Therefore, the contrast in HAADF-STEM imaging is lower compared with the contrast generated by metallic nanoparticles. In this way, the identification of metallic nanostructures embedded in biological tissue is relatively easy to observe. In the Fig. 20 silver nanoparticles are incorporated into bacteria and the particles can be clearly identified by contrast. HAADF-STEM imaging can avoid the staining procedure in comparison with the conventional TEM.

In summary, the scanning transmission electron microscope overcomes the barrier to atomic resolution, making it in one of the most powerful tools for characterization of nanomaterials. The new generation of aberration corrected microscopes, besides being a source of sophisticated characterization techniques, will certainly become a fundamental bridge between materials and health sciences. The capacity to control size, shape, and surface chemistry versatility of engineered nanoparticles has enabled their use into a plethora of biological and medical applications as drug delivery systems, therapeutics, diagnostics, and imaging contrast agents with different advanced functions and new properties. Depending of particular nanoparticle properties and mechanisms of interaction with biological systems, adsorption and uptake can produce dose-dependent decreases in cell viability and different metabolic and genetic alterations. These phenomena can be monitored with different well-established biochemical and molecular biology 
assays. The STEM methods mentioned in this chapter can be used to confirm attachment, internalization, and intracellular localization of these nanomaterials. As an example, cancer nanotechnology focuses on applications based on engineered nanoparticles designed for detection, diagnosis, targeting, and treatment of cancerous cells. Noble metal nanoparticles are biocompatible and non-toxic agents useful in all these biomedical applications at the cellular or molecular scale. Other important structural characteristics of nanoparticles for biological and biomedical applications that need to be determined are: size, shape, hydrodynamic radius, zeta potential, surface charge, defects, chemical composition, and atomic structure, that is highly related to functionality, stability, and reactivity of nanoparticles.

\section{Acknowledgements}

The authors would like to acknowledge THE WELCH FOUNDATION AGENCY PROJECT \# AX-1615. "Controlling the Shape and Particles Using Wet Chemistry Methods and Its Application to Synthesis of Hollow Bimetallic Nanostructures." The authors would also like to acknowledge the NSF PREM Grant \# DMR 0934218, Title: Oxide and Metal Nanoparticles-The Interface between life sciences and physical sciences. The authors would also like to acknowledge RCMI Center for Interdisciplinary Health Research CIHR. "The project described was supported by Award Number 2G12RR013646-11 from the National Center for Research Resources. The content is solely the responsibility of the authors and does not necessarily represent the official views of the National Center for Research Resources of the National Institutes of Health."

\section{References}

1. Pennycook S (1989) Z-contrast STEM for materials science. Ultramicroscopy 30:58-69

2. Ercolessi F, Andreoni W et al (1991) Melting of small gold particles: mechanism and size effects. Phys Rev Lett 66:911-914

3. Ferrando R, Jellinek J et al (2008) Nanoalloys: from theory to applications of alloy clusters and nanoparticles. Chem Rev 108:845-910

4. Carter CB, Williams DB (2009) Transmission electron microscopy: a textbook for materials science. Springer, New York

5. Fernández-Navarro C et al (2007) On the structure of $\mathrm{Au} / \mathrm{Pd}$ bimetallic nanoparticles. J Phys Chem C 111:1256-1260

6. Kirkland EJ (1998) Advanced computing in electron microscopy. Springer, New York
7. Zuo JM, Olsen E (2011) http://cbed.matse. illinois.edu/JProbe/JProbe.html. Accessed 12 Aug 2011

8. Wall J, Langmore J, Isaacson M et al (1977) Scanning transmission electron microscopy at high resolution. Proc Natl Acad Sci 74:1802-1806

9. Ishizuka K (1998) Multislice implementation for inclined illumination and convergent-beam electron diffraction. In: Shiojiri M \& Nishio K (eds) Proceedings of the International Symposium on Hybrid Analysis for Functional Nanostructure, Kyoto, Nakanishi Printing Co., pp 69-72

10. Pinker S (1997) How the mind works. W. Norton \& Company, New York 